\title{
Application of Ar Ion Beam Milling on Sectioning of Cells for SEM Observations
}

\author{
Shigeyasu Tanaka ${ }^{1}$ and Yusuke Ohmi ${ }^{1}$
}

1. College of Life and Health Science, Chubu University, Kasugai-shi, Aichi, Japan.

For cross-sectional observations of biological samples by electron microscopy, TEM is usually used. However, the sample preparation for TEM observations requires laborious procedures and takes from several hours to a day or more [1]. The sample preparation for SEM observations is less laborious, thus SEM is used more and more for cross-sectional observations. As a sectioning technique for biological samples, focused ion beam (FIB) has received much attention [2], because it has been used in material science and industry fields to obtain accurately and precisely sectioned samples. But, because of the high beam energy, ion-beam induced damage is more severe in the biological field, and precautions are vitally necessary. We developed an alternative technique for sectioning of cells, which is based on lowenergy Ar ion-beam milling, and is easy and rapid. Here, we report our method using a cultured cell (HEK293T) as a sample.

A silicon plate was coated by $0.1 \mathrm{mg} / \mathrm{mL}$ poly-L-lysine in phosphate buffered saline (PBS) for $3 \mathrm{~h}$ at room temperature. The coated silicon plate was washed by PBS. HEK293T cell $\left(1.4 \times 10^{6}\right.$ cell $\left./ \mathrm{ml}\right)$ was cultured with $10 \%$ FCS containing DMEM medium on the poly-L-lysine coated silicon plate overnight at $37^{\circ} \mathrm{C}$ in $5 \% \mathrm{CO}_{2}$ incubator. Then the cell on silicon was fixed with $3 \%$ glutaraldehyde solution for 2 h. After washing with PBS, the sample was stained with Platinum Blue. Then the sample was processed by BEL-1 (Nisshin EM). After this treatment, the sample was dried in a vacuum chamber. Then the silicon plate was cut into a rectangular shape (about $0.5 \mathrm{~mm} \times 3 \mathrm{~mm}$ ). In this process, sharp edges were created by cleavage. The sharp edge was used as a shield. Finally, the rectangular silicon piece was fixed to a sample holder of Ion Slicer (JEOL), and Ar ion beam was irradiated. The ion irradiation was carried out in such a way that most cells were located in the shadow of the silicon plate with respect to the Ar ion beam (Figure 1). Only upper portion of the cells near the edge of the silicon plate is sputtered. The position of sectioning can be controlled by changing the angle $\theta$ between the surface of the piece and the Ar ion beam. In this way, sectioned samples can be prepared easily. SEM observations were performed at an accelerating voltage of $3 \mathrm{kV}$.

Figure 2(a) shows a low-magnification SEM image of a sample prepared by this method. The cells are seen as dots on the Si plate. Figure 2(b) is a magnified image of a cell. The internal structure is clearly seen. The angle $\theta$ is about $20^{\circ}$ for this sample. Ar ion milling was carried out at an accelerating voltage of $3 \mathrm{kV}$ for $1 \mathrm{~h}$, followed $2 \mathrm{kV}$ cleaning process for $1 \mathrm{~h}$. The cleaning process using a low accelerating voltage was necessary to obtain good results. We used a cultured cell to demonstrate our method, but this method should be applicable to any type of biological samples.

References:

[1] G Knot and C Genoud, J. Cell Science 126 (2013), p. 4545.

[2] HL Hing et al, Microsc Microanal 13 (Suppl 2) (2007) p. 1528.

[3] This work was supported in part by JSPS KAKENHI Grant Number JP18K04246. 


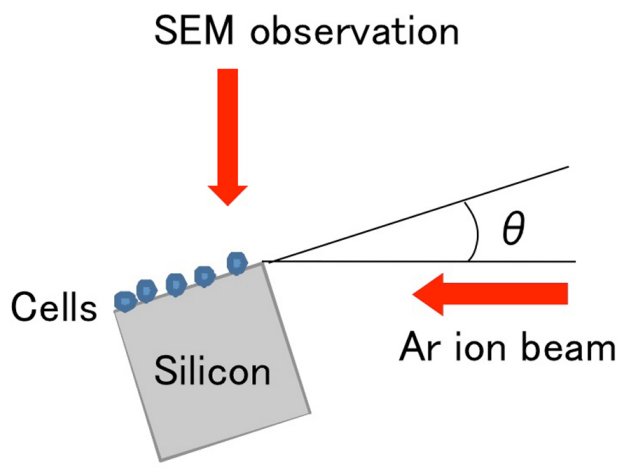

Figure 1. Schematic representation of sectioning process and SEM observation direction. Cells are on the Si surface and the Si surface is inclined with respect to the Ar ion beam. Most cells are located in the shadow of the silicon plate. Only upper portions of some cells near the edge are sputtered. The crosssection is very smooth and suitable for SEM observations.
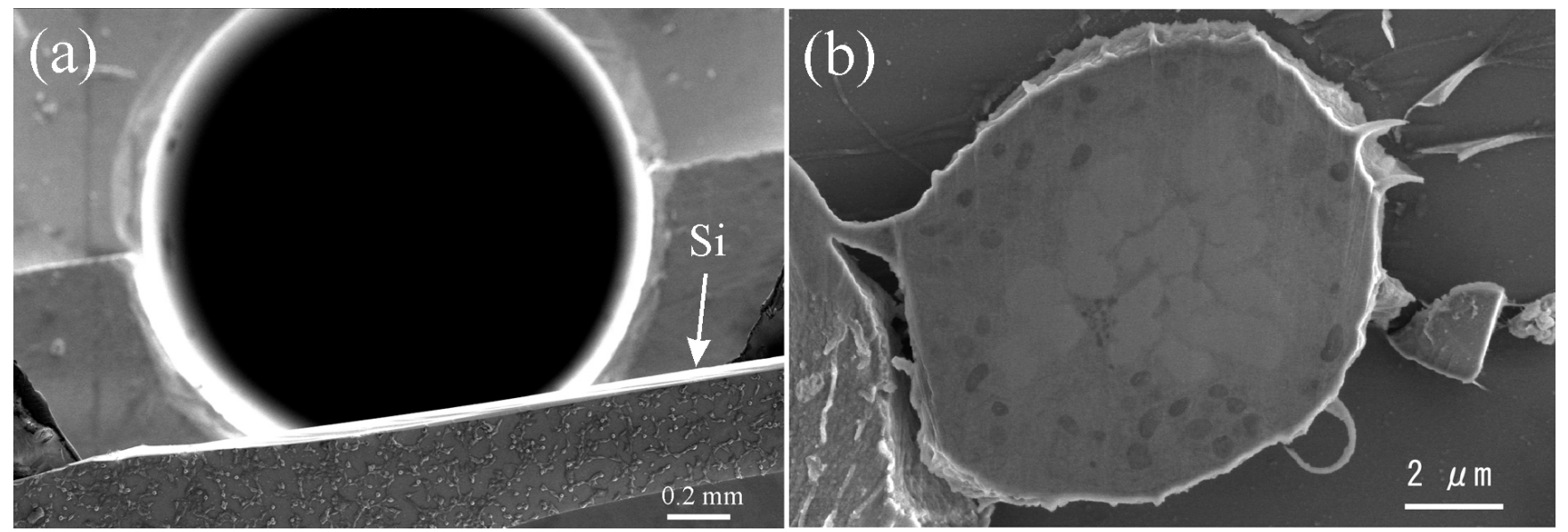

Figure 2. (a) Low-magnification SEM image of a sample prepared by this method. (b) Magnified image of a cell. The internal structure is clearly seen. 